\title{
ON UNIQUENESS IN GENERAL LINEAR VISCOELASTICITY*
}

\author{
BY \\ J. LUBLINER AND J. L. SACKMAN \\ University of California at Berkeley
}

Summary. On the basis of two theorems pertaining to the asymptotic behavior of certain Laplace transforms, the uniqueness of the displacement field in a general linear viscoelastic body (i.e., one with time-variable properties) throughout a time interval is demonstrated, provided the instantaneous elasticity tensor (or, in the case of a generalized Kelvin-Voigt material, the instantaneous viscosity tensor) is positive definite and a continuous function of time, and provided the following information is specified: the displacement field, to within a rigid-body motion, throughout the body and at all times before the given interval; the displacement and velocity fields throughout the body at the beginning of the interval (initial conditions); the body force throughout the body and throughout the interval; and, at each point of the boundary, in each of three orthogonal directions, a component of the traction or of the displacement throughout the time interval. If inertia is neglected, the initial conditions may be dispensed with, but the displacement field is unique only to within a rigid-body motion.

I. Introduction. A uniqueness theorem for the first boundary-value problem of the linear theory of viscoelasticity was apparently first established by V. Voltera [1], and was based on his earlier work on integro-differential equations [2]. This theorem applies to anisotropic, inhomogeneous, time-variable viscoelastic bodies with time-invariable instantaneous elastic response undergoing quasi-static deformations, but may be extended to include time-variable instantaneous elastic response.

Recently, Gurtin and Sternberg [3] called attention to Volterra's "little-known" but "remarkably strong uniqueness theorem". They slightly extended the theorem to accommodate stress-strain relations in creep-integral and differential-equation form, as well as in relaxation-integral form, restricting attention to isotropic, nonaging viscoelastic bodies.

In a subsequent paper, Sternberg and Gurtin [4] utilized the method developed by Volterra [1], [2] to extend Volterra's uniqueness theorem to thermo-rheologically simple, ablating viscoelastic solids undergoing quasi-static deformations. For such solids, the effective viscoelastic properties are time-variable due to the dependence of these propertics on the presence of a time-variable temperature field, but the instantaneous elastic response is taken to be time-invariable.

Prior to the restatement of Volterra's theorem by Gurtin and Sternberg [3], Breuer and Onat [5] established a uniqueness theorem for the mixed boundary-value problem of linear viscoelasticity theory. That theoicm deals with isotropic, non-aging viscoelastic bodies undergoing quasi-static deformations. It is less general than Volterra's theorem in still another respect: it requires that the relaxation functions be of "positive definite" type, whereas Volterra's theorem (interpreted for the isotropic case) requires only that the (time-invariable) instantaneous moduli of elasticity be positive.

*Received April 4, 1966; revised manuscript received July 8, 1966. 
Later, Onat and Breuer [6] generalized their uniqueness theorem so that, as in Volterra's theorem, positiveness of the instantaneous elastic shear and bulk moduli was required, rather than "positive definiteness" of the shear and bulk relaxation functions. Their proof of this theorem utilizes the theory of the Laplace transformation, which experience has shown to be a useful and natural tool in the analysis of problems of nonaging (i.e., time-invariable) linear viscoelasticity theory. The theorem was established for an isotropic, homogeneous, nonaging viscoelastic body undergoing quasi-static deformation under "mixed" boundary conditions. Various extensions to include inertia effects, anisotropy, and nonhomogeneity have been made by Edelstein and Gurtin [9] and Odeh and Tadjbakhsh [10].

Here we are concerned with a uniqueness theorem for a general linear viscoelastic body under "mixed-mixed" boundary conditions. By a general linear viscoelastic body we mean one which is anisotropic, nonhomogeneous and time-variable with respect to both instantaneous response and memory. Thus the theorem we establish is more general than the previously established uniqueness theorems for nonablating viscoelastic bodies.

In the construction of this theorem, we employ the Laplace transformation. Although the Laplace transformation is not as natural a tool of analysis for the general linear viscoelastic body as it is for the nonaging viscoelastic body, we find it useful in the development of the uniqueness theorem. By means of this transformation, and asymptotic methods, we are able to establish the positive-definiteness of a certain functional, from which uniqueness then follows directly.

II. Preliminary theorems. For any vector $\mathbf{u}$ with components $u_{i}(i=1, \ldots, n)$ we define the norm

$$
\|\mathbf{u}\|=\sum_{i=1}^{n}\left|u_{i}\right| .
$$

Similarly, for an $n \times n$ matrix A with elements $a_{i j}$ we define the norm

$$
\|\mathbf{A}\|=\sum_{i=1}^{n} \sum_{i=1}^{n}\left|a_{i j}\right| \text {. }
$$

For two vectors $\mathbf{u}, \mathbf{v}$ we define the inner product

$$
\mathbf{u} \cdot \mathbf{v}=\sum_{i=1}^{n} u_{i} v_{i} .
$$

The following inequalities are easily proved, and will be used in subsequent work:

$$
\begin{aligned}
\|\mathbf{u}+\mathbf{v}\| & \leq\|\mathbf{u}\|+\|\mathbf{v}\|, \\
\|\mathbf{A} \mathbf{u}\| & \leq\|\mathbf{A}\|\|\mathbf{u}\|, \\
|\mathbf{u} \cdot \mathbf{v}| & \leq\|\mathbf{u}\|\|\mathbf{v}\| .
\end{aligned}
$$

Another important inequality is

$$
\|\mathbf{u}\|^{2} \leq n \mathbf{u} \cdot \mathbf{u} .
$$

This follows from the Cauchy-Schwartz inequality,

$$
(\mathbf{u} \cdot \boldsymbol{v})^{2} \leq(\mathbf{u} \cdot \mathbf{u})(\boldsymbol{v} \cdot \boldsymbol{v}),
$$


if we set $v_{i}=\operatorname{sgn} u_{i}, i=1, \cdots, n$. For a vector function $\mathbf{u}(x)$ of a real variable $x$, defined over $a<x<b$, the following inequality holds:

$$
\left\|\int_{a}^{b} \mathbf{u}(x) d x\right\| \leq \int_{a}^{b}\|\mathbf{u}(x)\| d x .
$$

We shall now consider the asymptotic behavior of vector functions of $x$ as $x \rightarrow \infty$. We write

$$
\mathbf{u}(x)=O(\mathbf{v}(x)) \text { as } x \rightarrow \infty
$$

if there exists $x_{0}$ and a positive constant $A$ such that $\|\mathbf{u}\| \leq A\|\mathbf{v}\|$ for all $x>x_{0}$; and

$$
\mathbf{u}(x)=o(\mathbf{v}(x)) \text { as } x \rightarrow \infty
$$

if for any $\epsilon>0$ there exists an $x_{\epsilon}$ such that $\|\mathbf{u}\| \leq \epsilon\|\mathbf{v}\|$ for all $x>x_{\mathbf{e}}$. In (1.10) and (2.11) $\mathbf{u}$ and $\mathbf{v}$ need not have the same number of dimensions; in particular, one (or both) may be a scalar function, e.g., $\mathbf{u}(x)=O\left(e^{k x}\right)$. Furthermore, equivalent definitions apply to matrices.

The following little theorem will prove important later on:

If $\mathbf{u}(x)=o(\mathbf{v}(x))$ as $x \rightarrow \infty$, then $\mathbf{u}(x) \cdot \mathbf{v}(x)=o(\mathbf{v}(x) \cdot \mathbf{v}(x))$ as $x \rightarrow \infty$.

Proof. By (2.6), (2.7), and (2.11), $|\mathbf{u} \cdot \mathbf{v}| \leq\|\mathbf{u}\|\|\mathbf{v}\| \leq \epsilon\|\mathbf{v}\|^{2} \leq n \in \mathbf{v} \cdot \mathbf{v}$ for any $\boldsymbol{\epsilon}$, for $x>x_{\epsilon}$.

We shall next state and prove a lemma and two theorems pertaining to Laplace transforms of vector functions of $t$ defined over $0<t<\infty$. For any $\mathrm{f}(t)$ with components $f_{i}(t)$ the Laplace transform is

$$
\overline{\mathbf{f}}(s)=\mathscr{L}\{\mathbf{f}\}=\int_{0}^{\infty} e^{-s t} \mathbf{f}(t) d t
$$

with components

$$
\bar{f}_{i}(s)=\mathcal{L}\left\{f_{i}\right\}=\int_{0}^{\infty} e^{-s t} f_{i}(t) d t
$$

defined over $c<s<\infty, c$ being large enough to insure the convergence of the integrals $\left(2.13^{\prime}\right)$. Throughout the subsequent work, s will be treated as a real variable.

Lemma. If $\mathbf{h}(t)$ is a vector function possessing a Laplace transform $\mathbf{h}(s)$ which converges absolutely for $s$ sufficiently large, and there exist values $t_{0}$ and $t_{1}$, with $0 \leq t_{0} \leq t_{1}$, such that

(a) $\mathbf{h}(t)=\mathbf{0}$ for $t<t_{0}$,

(b) $\|\mathrm{h}(t)\|>0$ and none of the $h_{\mathbf{i}}(t)$ changes sign for $t_{0} \leq t \leq t_{1}$, then

$$
\mathscr{L}\{\|\mathbf{h}\|\}=\|\overline{\mathrm{h}}(s)\|+o\left(e^{-\Delta t_{1}}\right) \text { as } s \rightarrow \infty .
$$

Proof. Define a vector function $\mathrm{h}^{*}(t)$ as follows:

$$
\begin{aligned}
\mathrm{h}^{*}(t) & =\mathrm{h}(t), \quad t<t_{1}, \\
& =\mathrm{h}\left(t_{1}-\right), \quad t \geq t_{1} .
\end{aligned}
$$

The $h_{i}^{*}(t)$ do not change sign; hence

$$
\mathcal{L}\left\{\left\|\mathbf{h}^{*}\right\|\right\}=\left\|\overline{\mathbf{h}}^{*}(s)\right\| .
$$


Let

$$
\mathbf{u}(s) \equiv \overline{\mathbf{h}}^{*}(s)-\overline{\mathbf{h}}(s) .
$$

Clearly [7, p. 481]

$$
\mathbf{u}(s)=o\left(e^{-a t_{1}}\right) \text { as } s \rightarrow \infty .
$$

But

$$
\left\|\overline{\mathbf{h}}^{*}(s)\right\|-\|\overline{\mathbf{h}}(s)\| \leq\left\|\overline{\mathbf{h}}^{*}(s)-\overline{\mathrm{h}}(s)\right\|=\|\mathbf{u}(s)\|
$$

so that

$$
\left\|\overline{\mathrm{h}}^{*}(s)\right\|=\|\overline{\mathrm{h}}(s)\|+o\left(e^{-\mathrm{et} \mathrm{s}}\right) \text { as } s \rightarrow \infty
$$

Similarly,

$$
\mathscr{L}\left\{|| \mathbf{h}^{*} \|\right\}=\mathscr{L}\{|| \mathbf{h} \|\}+o\left(e^{-s t_{2}}\right) \text { as } s \rightarrow \infty .
$$

By combining (2.14), (2.15) and (2.16), we prove the Lemma.

On noting that, for any $a$ such that $t_{0} \leq a \leq t_{1}$,

$$
\lim _{a \rightarrow \infty} e^{s a}\|\overline{\mathbf{h}}(s)\|=\lim _{s \rightarrow \infty} e^{a a} \mathfrak{L}\{\|\mathbf{h}\|\},
$$

we can easily demonstrate the following

Corollary. If $t_{0}<a \leq t_{1}$, then

$$
\lim _{a \rightarrow \infty} e^{a}\|\overline{\mathrm{h}}(s)\|=\infty .
$$

Theorem 1. If $\mathbf{h}(t)$ satisfies the conditions of the Lemma, and $\mathrm{Q}(s, t)$ is a matrix function tending to zero as $s \rightarrow \infty$ uniformly in t, then

$$
\int_{0}^{\infty} e^{-\cdot t} \mathrm{Q}(s, t) \mathrm{h}(t) d t \equiv \mathbf{g}(s)=o(\overline{\mathbf{h}}(s)) \quad \text { as } \quad s \rightarrow \infty .
$$

Proof. For any $\epsilon>0$ there exists an $s_{0}$ independent of $t$, such that

$$
\|\mathrm{Q}(s, t)\|<\epsilon / 2 \text { for } s>s_{0} \text {; }
$$

hence, for $s>s_{0}$,

$$
\|\mathbf{g}(s)\|<(\epsilon / 2) \mathfrak{L}\{\mid \mathbf{h} \|\}
$$

From the Lemma we have

$$
\mathcal{L}\{\|\mathbf{h}\|\}=\|\overline{\mathbf{h}}(s)\|+o\left(e^{-t_{1}}\right) \text { as } s \rightarrow \infty .
$$

By the Corollary to the Lemma, the last term can be made less than $\|\overline{\mathbf{h}}(s)\|$ for $s$ sufficiently large; supposing $s_{0}$ to be large enough, we have

$$
\|\mathbf{g}(s)\|<\epsilon\|\overline{\mathrm{h}}(s)\| \text { for } s>s_{0},
$$

proving the theorem.

Theorem 2. If $\mathbf{h}(t)$ satisfies the conditions of the Lemma, and if

$$
\mathbf{f}(t)=\mathbf{G}(t) \mathbf{h}(t)
$$

where $\mathrm{G}(t)$ is a continuous matrix function, then, if $\overline{\mathrm{f}}(s)$ exists, 


$$
\overline{\mathrm{f}}(s)=\mathbf{G}\left(t_{0}\right) \overline{\mathrm{h}}(s)+o(\overline{\mathrm{h}}(s)) \quad \text { as } s \rightarrow \infty .
$$

Proof. Since $\mathrm{G}(t)$ is continuous, for any $\epsilon>0$ there exists an $a>t_{0}$ such that

$$
\left\|\mathbf{G}(t)-\mathbf{G}\left(t_{0}\right)\right\|<\epsilon / 4 \text { for } t_{0} \leq t \leq a .
$$

In particular, we may take $a \leq t_{1}$. We now write

$$
\begin{aligned}
\mathbf{u}(s) & \equiv \overline{\mathbf{f}}(s)-\mathbf{G}\left(t_{0}\right) \overline{\mathbf{h}}(s) \\
& =\mathbf{u}^{*}(s)+o\left(e^{-s a}\right) \text { as } s \rightarrow \infty,
\end{aligned}
$$

where

$$
\mathbf{u}^{*}(s)=\int_{0}^{a}\left[\mathbf{G}(t)-\mathbf{G}\left(t_{0}\right)\right] \mathbf{h}(t) e^{-s t} d t .
$$

From the above definition,

$$
\left\|\mathbf{u}^{*}(s)\right\|<(\epsilon / 4) \int_{0}^{a}\|\mathrm{~h}(t)\| e^{-s t} d t \leq(\epsilon / 4) \mathscr{L}\{\|\mathbf{h}\|\} .
$$

As in Theorem 1, we may show

$$
\left\|\mathbf{u}^{*}(s)\right\| \leq(\epsilon / 2)\|\overline{\mathbf{h}}(s)\|
$$

for $s$ sufficiently large. Furthermore, by the Corollary to the Lemma we can find $s$ sufficiently large so that the second term of $(2.17)$ is less than $(\epsilon / 2)\|\overline{\mathbf{h}}(s)\|$. Consequently there exists an $s_{0}$ such that

$$
\|\mathbf{u}(s)\|<\epsilon \mid\|\overline{\mathbf{h}}(s)\| \text { for } s>s_{0} .
$$

The theorem is thus proved.

\section{The uniqueness principle.}

3.1 Description of a viscoelastic body. Consider a viscoelastic body occupying a closed region $R$ in three-dimensional space; the boundary of $R$ is $B$. We are concerned with infinitesimal deformations of the body, i.e., deformations so small that the body may be regarded as occupying $R$ throughout its history. If we use Cartesian coordinates $x_{i}(i=1,2,3)$ to denote a point $x$ of $R$, and $t$ to denote time, then the mechanical state of the body is specified by the displacement vector $\mathbf{u}(x, t)$ with components $u_{i}(x, t)$, the strain tensor $\varepsilon(x, t)$ with components $\epsilon_{i j}(x, t)$ and the stress tensor $\boldsymbol{\delta}(x, t)$ with components $\sigma_{i j}(x, t)$; the indices $i, j$ range over $1,2,3$. The strain components are given by

$$
\epsilon_{i j}=\frac{1}{2}\left(u_{i, i}+u_{i, i}\right)
$$

where

$$
()_{, i} \equiv \frac{\partial}{\partial x_{i}}()
$$

The stress tensor is symmetric and satisfies the equation of motion

$$
\sigma_{i j, i}+f_{i}(x, t)=\rho(x) \ddot{u}_{i}
$$

where $(\cdot) \equiv \partial / \partial t(), f$ is the body force, and $\rho$ is the density in the rest state.

At a point $x$ of $B$, if $\mathbf{n}$ denotes the outward unit normal vector, then the traction 
vector $t$ is defined by

$$
t_{i}=n_{j} \sigma_{i j} .
$$

To express the relation between stress and strain, it is convenient to represent them as vectors in 6 -space, with components $\sigma_{\alpha}, \epsilon_{\alpha}(\alpha=1, \cdots, 6)$. The constitutive equation of general linear viscoelasticity takes the form

$$
\boldsymbol{\delta}(x, t)=\mathbf{H}(x, t) \dot{\varepsilon}(x, t)+\int_{-\infty}^{t} \mathbf{G}(x, t, \tau) \dot{\varepsilon}(x, \tau) d \tau,
$$

where $\mathrm{H}$ and $\mathrm{G}$ are matrices with elements $H_{\alpha \beta}, G_{\alpha \beta}(\alpha, \beta=1, \cdots, 6) . \mathrm{H}$ represents initial Newtonian viscosity, and $\mathrm{G}$ represents elasticity and relaxation; Equation (3.4) represents, therefore, the behavior of a generalized Kelvin-Voigt material. If $\mathbf{H}=\mathbf{0}$ then the constitutive equation

$$
\boldsymbol{\delta}(x, t)=\mathbf{E}(x, t) \boldsymbol{\varepsilon}(x, t)-\int_{-\infty}^{t} \mathbf{R}(x, t, \tau) \boldsymbol{\varepsilon}(x, \tau) d \tau
$$

where $\mathbf{E}(x, t)=\mathbf{G}(x, t, t)$ and $\mathbf{R}=(\partial / \partial \tau) \mathbf{G}$, may be obtained from (3.4) by integration by parts. E represents instantaneous elasticity, and R "memory" or "heredity". Equation (3.5) corresponds essentially to Volterra's "hereditary elasticity", except that Volterra assumed $\mathrm{E}$ independent of $t$. In the subsequent work we shall treat viscoelastic materials governed by (3.5) though ultimately we shall direct attention to generalized Kelvin-Voigt materials as well.

3.2 Histories. By a history $\mathcal{H}(t)$ we shall mean a vector function $\mathbf{u}(x, \tau)$ defined for all $x$ in $R$ and $\tau<t$, and the corresponding functions $\varepsilon$ and $\boldsymbol{\sigma}$ given by (3.1) and (3.5). A vector function $\mathbf{f}(x, \tau)$ is associated with the history, and related to it by (3.2). By a null history $\mathcal{O}(t)$ we shall mean a function $\mathbf{u}(x, \tau)$ such that $\boldsymbol{\varepsilon}$ (and hence $\boldsymbol{\delta}$ ) vanishes for all $x$ in $R$ and $\tau<t$ (i.e., a rigid-body motion). For two histories $\mathfrak{F}_{1}(t)$ and $\mathfrak{H C}_{2}(t)$ we shall define their sum and difference $\mathfrak{F C}_{1}(t) \pm \mathcal{K}_{2}(t)$ by the vector functions $\mathbf{u}_{1}(x, \tau) \pm$ $\mathbf{u}_{2}(x, \tau)$; since the operations in (3.1) and (3.5) are linear, we also have the corresponding functions $\varepsilon_{1} \pm \varepsilon_{2}$ and $\sigma_{1} \pm \sigma_{2}$. We shall call $\mathfrak{K}_{1}(t)$ and $\mathcal{H}_{2}(t)$ equivalent, and write $\mathfrak{F C}_{1}(t) \doteq \mathfrak{F C}_{2}(t)$, if $\mathfrak{F}_{1}(t)-\mathfrak{F C}_{2}(t)=\mathcal{O}(t)$.

A non-null history $\mathcal{H}(t)$ will be considered admissible if the function $\varepsilon$ satisfies the following two conditions:

(1) It is infinitesimal [8, p. 242];

(2) Any time interval may be divided into a finite number of intervals in which none of the $\epsilon_{\alpha}$ changes sign (i.e., has both positive and negative values).

Condition (2) excludes, for example, time functions of the type sin $(1 / t)$. Condition (1) requires $\mathbf{u}$ to be a continuous function of $x$. No further continuity condition will be imposed $a$ priori; it will be assumed that any discontinuities will be such that the governing equations can be satisfied in the distribution sense. Attention will be drawn to the fact that the continuity requirements of Refs. 3 and 5 exclude shock waves.

3.3 Uniqueness. We shall now consider two admissible histories $\mathfrak{H C}_{1}(t)$ and $\mathfrak{H C}_{2}(t)$, with $t>0$, such that

$$
\begin{array}{r}
\mathcal{H C}_{1}(0) \doteq \mathcal{F C}_{2}(0) ; \\
\mathbf{u}_{1}(x, 0)=\mathbf{u}_{2}(x, 0), \quad x \varepsilon R ;
\end{array}
$$




$$
\begin{aligned}
\dot{\mathfrak{u}}_{1}(x, 0) & =\dot{\mathfrak{u}}_{2}(x, 0), \quad x \varepsilon R ; \\
\mathrm{f}_{1}(x, \tau)=\mathrm{f}_{2}(x, \tau), & x \varepsilon R, \quad 0<\tau<t ;
\end{aligned}
$$

and

$$
t_{1 k}(x, \tau)=t_{2 k}(x, \tau) \text { or } u_{1 k}(x, \tau)=u_{2 k}(x, \tau)
$$

for each $k(k=1,2,3)$ referred to a local orthogonal base at $x, x \varepsilon B, 0<\tau<t$. If we define a history

$$
\mathfrak{H C}^{\prime}(t)=\mathfrak{H}_{1}(t)-\mathfrak{H}_{2}(t)
$$

i.e., $\mathbf{u}^{\prime}=\mathbf{u}_{1}-\mathbf{u}_{2}$, etc., then

$$
\begin{aligned}
\mathcal{F}^{\prime}(0) & =\mathcal{O}(0) ; \\
\mathfrak{u}^{\prime}(x, 0) & =0 ; \\
\dot{\mathfrak{u}}^{\prime}(x, 0) & =0 ; \\
t_{k}^{\prime}(x, \tau) & =0 \text { or } u_{k}^{\prime}(x, \tau)=0
\end{aligned}
$$

for each $k$ as before, $x \varepsilon B, 0<\tau<t$.

As a consequence of (3.11) we have

$$
\boldsymbol{\sigma}^{\prime}(t)=\mathbf{E}(t) \mathbf{\varepsilon}^{\prime}(t)-\int_{0}^{t} \mathbf{R}(t, \tau) \varepsilon^{\prime}(\tau) d \tau,
$$

with the dependence on $x$ not explicitly indicated but to be understood henceforth. We also have, by virtue of (3.9),

$$
\sigma_{i, j}^{\prime}=\rho \ddot{u}_{i}^{\prime} .
$$

We now introduce Laplace transforms of the functions $\mathbf{u}^{\prime}, \mathbf{\varepsilon}^{\prime}, \boldsymbol{\sigma}^{\prime}, \mathbf{t}^{\prime}$, to $b=$ denoted by $\overrightarrow{\mathbf{u}}^{\prime}(s)$, etc. We form the surface integral

$$
I=\int_{B} \overline{\mathfrak{t}}^{\prime}(s) \cdot \overline{\mathbf{u}}^{\prime}(s) d s,
$$

which, by (3.14), must vanish for all $s$. By means of the divergence theorem we obtain

$$
\begin{aligned}
I & =\int_{B} n_{i} \bar{\sigma}_{i j}^{\prime}(s) \bar{u}_{i}^{\prime}(s) d s \\
& =\int_{R}\left[\bar{\sigma}_{i j}^{\prime}(s) \bar{u}_{i}^{\prime}(s)\right]_{, i} d V \\
& =I_{1}+I_{2}
\end{aligned}
$$

where

$$
\begin{aligned}
I_{1} & -\int_{R} \bar{\sigma}_{i, j}^{\prime}(s) \bar{u}_{i}^{\prime}(s) d V \\
& =\int_{R} \rho s^{2} \overline{\mathbf{u}}^{\prime}(s) \cdot \overline{\mathbf{u}}^{\prime}(s) d V
\end{aligned}
$$

by (3.12), (3.13), and (3.16), and 


$$
\begin{aligned}
I_{2} & =\int_{R} \bar{\sigma}_{i j}^{\prime}(s) \bar{u}_{i, j}^{\prime}(s) d V \\
& =\int_{R} \overline{\mathbf{\sigma}}^{\prime}(s) \cdot \overline{\boldsymbol{\varepsilon}}^{\prime}(s) d V
\end{aligned}
$$

by (3.1) and the symmetry of 8 .

It is clear that $I_{1}$ cannot be negative, and will vanish if and only if $\overline{\mathbf{u}}^{\prime}(s)$ vanishes everywhere in $R$. We now proceed to study $I_{2}$. We write

$$
\boldsymbol{\delta}^{\prime}=\boldsymbol{d}^{\prime \prime}-\boldsymbol{\delta}^{\prime \prime \prime},
$$

where

$$
\boldsymbol{\sigma}^{\prime \prime}(t)=\mathbf{E}(t) \mathbf{\varepsilon}^{\prime}(t)
$$

and

$$
\mathbf{\sigma}^{\prime \prime \prime}(t)=\int_{0}^{t} \mathbf{R}(t, \tau) \varepsilon^{\prime}(\tau) d \tau
$$

If $\mathcal{H C}^{\prime}(t)$ is not a null history, then there must be a subset $Q$ of $R$ in which $\varepsilon^{\prime}$ does not vanish identically for $0<\tau<t$. Furthermore, $\mathcal{H}^{\prime}(t)$ is admissible; hence, for each point $x$ of $Q$ there exists an interval $t_{0}<\tau<t_{1}$, with $0 \leq t_{0}<t_{1} \leq t$ such that $\boldsymbol{\varepsilon}^{\prime}(\tau)=0$ for $\tau<t_{0}$, while in $t_{0}<\tau<t_{1}$, at least one of the $\epsilon_{\alpha}$ does not vanish (so that $\|\varepsilon\|>0$ ) and none of them change sign. Since $\varepsilon^{\prime}$ is bounded, $\bar{\varepsilon}^{\prime}(s)$ converges absolutely for $s>0$ [7, p. 33]. Consequently $\varepsilon^{\prime}$ satisfies the condition of the Lemma of Sec. 2. Then, by Theorem 2.

$$
\overline{\boldsymbol{\sigma}}^{\prime \prime}(s)=\mathbf{E}\left(t_{0}\right) \overline{\boldsymbol{\varepsilon}}^{\prime}(s)+o\left(\overline{\boldsymbol{\varepsilon}}^{\prime}(s)\right) \text { as } s \rightarrow \infty .
$$

The Laplace transform of $\boldsymbol{o}^{\prime \prime \prime}$ is

$$
\begin{aligned}
\overline{\mathbf{\delta}}^{\prime \prime \prime}(s) & =\int_{0}^{\infty} e^{-s t} \int_{0}^{t} \mathbf{R}(t, \tau) \varepsilon^{\prime}(\tau) d \tau d t \\
& =\int_{0}^{\infty} e^{-s \tau}\left[\int_{0}^{\infty} e^{-s \tau^{\prime}} \mathbf{R}\left(\tau+\tau^{\prime}, \tau\right) d \tau^{\prime}\right] \boldsymbol{\varepsilon}^{\prime}(\tau) d \tau ;
\end{aligned}
$$

but the quantity in brackets is the Laplace transform with respect to $\boldsymbol{\tau}^{\prime}$ of $\mathbf{R}\left(\boldsymbol{\tau}+\boldsymbol{\tau}^{\prime}, \tau\right)$ and therefore vanishes as $s \rightarrow \infty$ uniformly in $\tau$ [7, p. 162]. Consequently, by Theorem 1 ,

$$
\overline{\mathbf{d}}^{\prime \prime \prime}(s)=o\left(\overline{\boldsymbol{\varepsilon}}^{\prime}(s)\right) \text { as } s \rightarrow \infty
$$

and therefore

$$
\overline{\mathbf{d}}^{\prime}(s)=\mathrm{E}\left(t_{0}\right) \overline{\boldsymbol{\varepsilon}}^{\prime}(s)+o\left(\overline{\boldsymbol{\varepsilon}}^{\prime}(s)\right) \text { as } s \rightarrow \infty .
$$

Lastly, by (2.12)

$$
\overline{\mathbf{d}}^{\prime}(s) \cdot \overline{\boldsymbol{\varepsilon}}^{\prime}(s)=\overline{\boldsymbol{\varepsilon}}^{\prime}(s) \cdot \mathbf{E}\left(t_{0}\right) \overline{\boldsymbol{\varepsilon}}^{\prime}(s)+o\left(\overline{\boldsymbol{\varepsilon}}^{\prime}(s) \cdot \overline{\boldsymbol{\varepsilon}}^{\prime}(s)\right) \text { as } s \rightarrow \infty .
$$

If $\mathbf{E}\left(t_{0}\right)$ is a positive definite matrix (i.e., has only positive real eigenvalues), then

$$
\overline{\boldsymbol{\varepsilon}}^{\prime}(s) \cdot \mathbf{E}\left(t_{0}\right) \overline{\boldsymbol{\varepsilon}}^{\prime}(s) \geq E_{1} \overline{\boldsymbol{\varepsilon}}^{\prime}(s) \cdot \overline{\boldsymbol{\varepsilon}}^{\prime}(s),
$$

where $E_{1}$ is the smallest eigenvalue of $\mathbf{E}\left(t_{0}\right)$. Hence the second term on the right of (3.22) 
can be made numerically less than the first for $s$ sufficiently large. Therefore there exists an $s_{x}$ such that $\overline{\boldsymbol{\sigma}}^{\prime}(s) \cdot \overline{\boldsymbol{\varepsilon}}^{\prime}(s)$ must be positive for $s \geq s_{x}$. Defining

$$
s_{0}=\max _{x=0} s_{x}
$$

We conclude that $I_{2}$ must be positive for $s>s_{0}$. Since, however, $I_{1}$ cannot be negative, $I$ cannot vanish for $s>s_{0}$. We have therefore contradicted the assumption that $\mathcal{F}^{\prime}(t)$ is not a null history.

If $\mathfrak{H C}^{\prime}(t)$ is a null history, then, $I_{2}$ vanishes, and $I$ equals $I_{1} . I$ can therefore vanish if and only if $\tilde{\mathbf{u}}^{\prime}(s)$ vanishes everywhere in $R$ and, consequently, if and only if $\mathbf{u}^{\prime}(x, \tau)$ vanishes everywhere in $R, 0<\tau<t$.

We have thus proved that for two admissible histories $\mathfrak{F C}_{1}(t)$ and $\mathfrak{F C}_{2}(t)$ related by (3.6)-(3.10), the displacement (and hence the strain and stress) fields are equal in the time interval $0<\tau<t$ for a viscoelastic body governed by (3.5) if $\mathrm{E}(x, \tau)$ is a positive definite matrix and a continuous function of $\tau$ in $0<\tau<t$.

3.4 Extension to generalized Kelvin-Voigt material. There is no evidence that any real materials are of the generalized Kelvin-Voigt type, i.e., behave instantaneously like Newtonian fluids; even water possesses some instantaneous shear elasticity. ${ }^{1}$ Nevertheless, for the sake of theoretical interest, we shall show how the uniqueness theorem enunciated in the previous subsection may be extended to such materials. If we form, instead of $I$, the integral ${ }^{2}$

$$
I^{\prime}=\int_{B} \overline{\mathfrak{t}}^{\prime}(s) \cdot \overline{\mathbf{v}}^{\prime}(s) d S,
$$

then, on noting the condition (3.7), we see immediately that $I^{\prime}=s I$; by an analogous definition $I_{1}^{\prime}=s I_{1}$ and $I_{1}^{\prime} \geq 0$ for $s>0$. The corresponding integral $I_{2}^{\prime}$ ha the integrand $\overline{\mathbf{d}}^{\prime}(s) \cdot \overline{\mathbf{n}}^{\prime}(s)$, and this can, by complete analogy with the procedure of $3.3,{ }^{3}$ he shown to be positive for large $s$ unless $\overline{\mathbf{n}}^{\prime}$ (and hence $\overline{\boldsymbol{\varepsilon}}^{\prime}$ ) vanishes, provided $\mathbf{H}(t)$ is continuous and positive definite. This uniqueness criterion is much weaker than the one enunciated for generalized Kelvin-Voigt materials by Gurtin and Sternberg, [3, Theorem 8.2 (b)].

IV. Additional remarks.

4.1. Isotropic materials. If a viscoelastic material governed by (3.5) is isotropic in regard to instantaneous behavior, the $\mathrm{E}$ matrix has the form

$$
\mathrm{E}=\left[\begin{array}{cccccc}
G_{1}+\frac{4}{3} G_{2} & G_{1}-\frac{2}{3} G_{2} & G_{1}-\frac{2}{3} G_{2} & 0 & 0 & 0 \\
& G_{1}+\frac{4}{3} G_{2} & G_{1}-\frac{2}{3} G_{2} & 0 & 0 & 0 \\
& & G_{1}+\frac{4}{3} G_{2} & 0 & 0 & 0 \\
& & & 2 G_{2} & 0 & 0 \\
& & & & 2 G_{2} & 0 \\
& & & & & 2 G_{2}
\end{array}\right]
$$

where $G_{1}$ is the (time-variable) instantaneous "bulk modulus" and $G_{2}$ is the "shear

${ }^{1}$ See also [11, remark at the end of Sec. 5].

'In what follows, we use $\mathbf{v}$ for $\dot{\mathfrak{u}}$ and $\mathfrak{n}$ for $\dot{\boldsymbol{\varepsilon}}$.

'Except that $n$ must possess, for large $s$, an absolutely convergent Laplace transform. 
modulus". The eigenvalues are $3 G_{1}$ and (repeated fivefold) $2 G_{2}$. Hence, the condition for positive-definiteness of $\mathrm{E}$ is $G_{1}>0, G_{2}>0$.

A similar result will apply to the generalized Kelvin-Voigt material.

4.2. Quasi-static uniqueness. We may neglect the inertia of the macerial by letting $\rho$ vanish; then $I_{1}$ vanishes identically, and $I$ vanishes if and only if $I_{2}$ does, i.e., if $\mathcal{F}^{\prime}(t)$ is a null history. In a quasi-static treatment, therefore, the displacement field is unique only to within a rigid-body motion (as in Ref. [3]). Also, the initial conditions (3.7), (3.8) may be dispensed with.

V. Acknowledgement. This research was supported by the U. S. Army Research Office-Durham.

\section{REFERENCES}

1. V. Volterra, Sulle equazioni integro-differenziali della teoria dell' elasticita, Atti Accad. Lincei (5) 18, 296-301 (1909)

2. V. Volterra, Sulle equazioni integro-differenziali, Atti Accad. Lincei (5) 18, 167-174 (1909)

3. M. E. Gurtin and E. Sternberg, On the linear theory of viscoelasticity, Arch. Rat. Mech. Anal. 11, 291-356 (1962)

4. E. Sternberg and M. E. Gurtin, Uniqueness in the theory of thermo-rheologically simple ablating viscoelastic solids, Progress in Applied Mechanics, The Prager Anniversary Volume, The Macmillan Company, New York, 1963, pp. 373-384

5. S. Breuer and E. T. Onat, On uniqueness in linear viscoelasticity, Q. Appl. Math. 19, 355-359 (1962)

6. E. T. Onat and S. Breuer, On uniqueness in linear viscoelasticity, Progress in Applied Mechanics, The Prager Anniversary Volume, MacMillan Company, New York, 1963, pp. 349-353

7. G. Doetsch, Handbuch der Laplace-Transformation, Vol. I, Verlag Birkhäuser, Basel, 1950

8. B. D. Coleman and W. Noll, Foundations of linear viscoelasticity, Rev. Mod. Phys. 33, 239-249 (1961)

9. W. S. Edelstein and M. E. Gurtin, Uniqueness theorems in the linear theory of anisotropic viscoelastic solids, Arch. Rat. Mech. Anal. 17, 47-60 (1964)

10. F. Odeh and I. Tadjbakhsh, Uniqueness in the linear theory of viscoelasticity, Arch. Rat. Mech. Anal. 18, 244-250 (1965)

11. B. D. Coleman, On thermodynamics, strain impulses, and linear viscoelasticity, Arch. Rat. Mech. Anal. 17, 230-254 (1964) 